\title{
Expression and Significance of TWSG1 in Craniofacial Fibrous Dysplasia
}

Y Yi, H Yang, X Xu, J Hu

\begin{abstract}
Objective: This study aims to determine the expression and significance of Triple Wide Straight Grooves 1

(TWSG1) in craniofacial fibrous dysplasia (FD).

Methods: TWSG1 expression was examined in specimens obtained from 44 patients with FD by immunohistochemistry.

Results: TWSG1 expression was positive in all FD patients, which was higher than in patients with normal mandible in the negative control group $(\mathrm{P}<0.05)$. Furthermore, the positive rate of TWSG1 was slightly higher than in adult patients with FD $(\mathrm{P}<0.05)$, which was classified by post-operativerecurrence or no recurrence. Moreover, the positive expression of TWSG1 in FD with favorable prognosis was higher than that with poor prognosis $(\mathrm{P}<0.05)$.
\end{abstract}

Conclusion: The overexpression of TWSG1 is a vital biological marker in the prognosis of FD.

Keywords: Fibrous dysplasia, immunohistochemistry, twisted gastrulation

From: ${ }^{1}$ Department of Stomatology, Quzhou People's Hospital, Zhejiang Province, Quzhou 324000, ${ }^{2}$ Department of Stomatology, Tenth People's Hospital, Tongji University School of Medicine, Shanghai 200072, ${ }^{3}$ Department of Stomatology, Quzhou People's Hospital, Zhejiang Province, Quzhou 324000, ${ }^{4}$ Jingzhou Hu. Department of Oral and Maxillofacial-Head and Neck Oncology, Ninth People's Hospital, Shanghai Jiao Tong University School of Medicine, Shanghai Key Laboratory of Stomatology and Shanghai Research Institute of Stomatology, Shanghai, 200011, China.

Correspondence: Dr J Hu, Department of Oral and Maxillofacial-Head and Neck Oncology

Ninth People's Hospital, Shanghai Jiao Tong University School of Medicine, Shanghai, 200011, China, E-mail: hujingzhou_h@163.com 


\section{INTRODUCTION}

Fibrous dysplasia (FD) is a congenital non-genetic bone tissue disorder that usually occurs in the craniofacial region. It can be self-limiting in adulthood, the average age of onset is low (1). and it can also be found in adults. The growth speed of FD is relatively fast. It causes deformities, headaches or other symptoms; and it affects the appearance. Its pathogenesis remains unclear. In recent years, studies have shown that TWSG1 plays an important role in the processes of bone formation and differentiation $(2,3)$. Through immunohistochemistry, this study investigated changes in TWSG1 expression in FD, and analyzed the relationship between positive expression and FD patients' ages, as well as between positive expression and other clinical prognostic factors. Finally, the effects of TWSG1 expression in FD were explored in the process and prognosis of the disease.

\section{MATERIALS AND METHODS}

\section{Tissue samples}

Forty-four craniofacial FD specimens confirmed by histopathology in the First Affiliated Hospital of Nanchang University from 2006 to 2012 were selected, including 21 cases of teenagers(under 18 years) and 23 cases of adults (18 years and above). Paraffin-embedded specimens were extracted to be sliced again. Samples were collected in the border of the lesion and normal bone tissue, and were stained with immunohistochemistry. In addition, these 44 specimens were divided into two groups according to postoperative recurrence. Furthermore, these specimens were further divided into two other groups according to the age 
of patients (above or below 18 years). The expression of TWSG1was compared among these groups. Ten cases of normal tissues from a fresh mandibular fracture in the Department of Stomatology of the First Affiliated Hospital of Nanchang University were taken as negative control. Patients, from which specimens were taken, were informed and agreed to participate in the study.

\section{Immunohistochemistry}

The primary antibody used was mouse anti-human monoclonal antibody TWSG1 (Taiwan Abnova Corporation), and the working titer was 1:500. Sections were made in water bath, deparaffinized and hydrated. Slice thickness was $3-4 \mu \mathrm{m}$. After antigen retrieval by microwave heating, sections were cooled to room temperature. Then, sections were washed three times in PBS for 3-5 minutes each time. Subsequently, cells were protected in $10 \%$ calf serum for 10 minutes to prevent nonspecific staining. After the serum was tossed away, the primary antibody was added. The sections were placed in a refrigerator overnight at $4{ }^{\circ} \mathrm{C}$, washed three times in PBS for three minutes each time, and colored by DAB color reagent. They were counterstained with hematoxylin for five minutes, made transparent in xylene, and mounted in gum.

\section{Interpretation of Results}

TWSG1 positive staining was mainly located in the cytoplasm. The cytoplasm, which presented as color yellow or brown, was considered to be positive. Cytoplasm without a 
yellow dye was considered to be negative. Labeled cells were manually counted. Five horizons of the FD lesion at high magnification $(\times 400)$ were randomly selected in order to count the positive stained cells. According to the percentage of positive cells, results were scored as follows: (zero) no positive cells; ( 1 point) <25\% positive cells; ( 2 points) $25-50 \%$ positive cells; ( 3 points) $51-75 \%$ positive cells; ( 4 points ) $>75 \%$ positive cells. Then, these results were scored according to staining intensity: (zero) negative, no colored cells; (1 point) weak positive cells with a light yellowish coloring; ( 2 points) positive cells that show a relatively obvious brown coloring; (3 points) strong positive cells that show brown coloring. The score of positive marks equals the intensity grade rating of positive stained cells, which is then multiplied by the number of positive stained cells. According to the results of the positive marks, results were divided into four levels: $0(-), 1$ to $2(+), 3$ to $4(++), 5$ to $9(+++)$; in which results no less than ++ was considered to be a strong expression.

\section{Statistical analysis}

Experimental data were treated with rank-sum test using SPSS19.0. $P<0.05$ was considered statistically significant.

\section{RESULTS}

\section{Positive expression of TWSG1 (44/44) in craniomaxillofacial fibrous dysplasia}

Brown particles in the cytoplasm/membrane are shown in the following figures, which are mainly observed in osteoblasts and fibroblasts (Figs. 1 and 2), in contrast with the expression 
of TWSG1in normal bone tissues (Fig. 3).

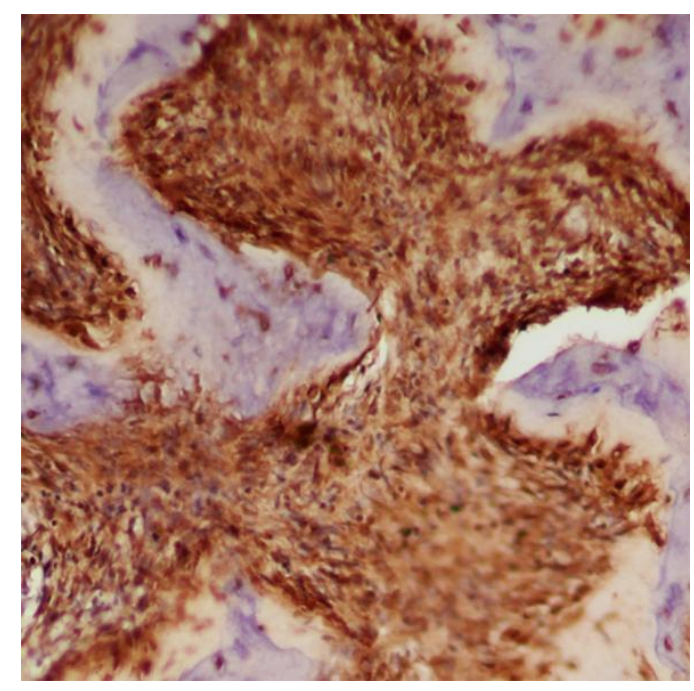

Fig. 1: Strong TWSG1 expression in osteoblasts and fibroblasts in FD $(\times 400)$.

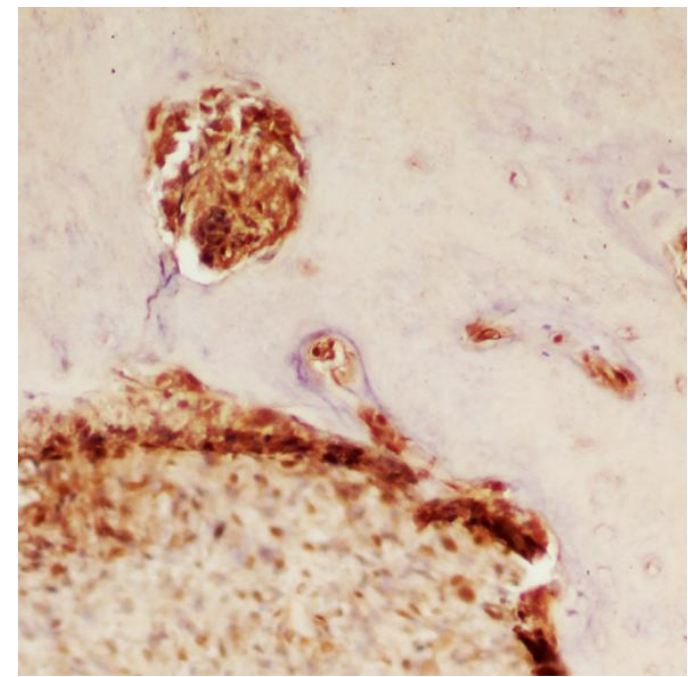

Fig. 2: Strong TWSG1 expression in fibroblasts in FD $(\times 400)$. 


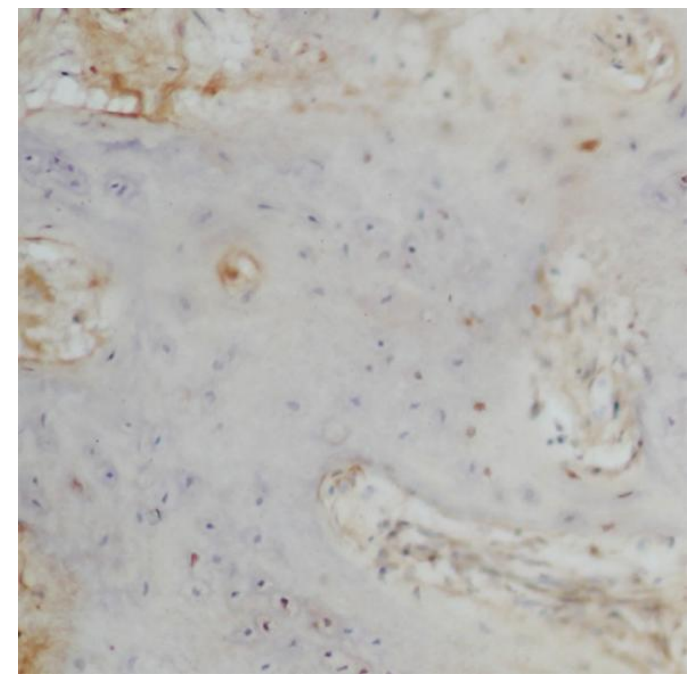

Fig 3: WeakTWSG1 expression in normal bone tissue $(\times 400)$.

The relationship between TWSG1 expression levels in FD tissue and ages, and TWSG1 expression in FD tissues and prognosis

The relationship between TWSG1 expression in FD tissues and ages, as well as TWSG1 expression in FD tissues and prognosis, are shown in Table 1. TWSG1 expression in FD patients were all positive and significantly higher than patients with normal mandible in the negative control group $(P<0.05)$. TWSG1 expression in minor FD patients were obviously higher than the adult groups $(P<0.05)$. Results were classified into groups according to whether or not recurrence occurred. TWSG1 expression in FD patients with good prognosis were obviously lower than patients with poor prognosis $(P<0.05)$. 
Table 1: The relationship between TWSG1 expression levels in FD tissues and ages, and TWSG1 expression in FD tissues and prognosis

\begin{tabular}{llllll}
\hline & $\begin{array}{l}\text { Negativ } \\
\mathrm{e}\end{array}$ & $\begin{array}{l}\text { Weak } \\
\text { positive }\end{array}$ & Positive & $\begin{array}{l}\text { Strong } \\
\text { positive }\end{array}$ & $\mathrm{P}$ \\
\hline $\begin{array}{l}\text { Teenagers } \\
\text { under } 18\end{array}$ & 0 & 4 & 12 & 5 & \\
$\begin{array}{l}\text { Adults } \\
\text { above } 18\end{array}$ & 0 & 2 & 13 & 8 & 0.008 \\
$\begin{array}{l}\text { Normal } \\
\text { groups }\end{array}$ & 8 & 2 & 0 & 0 & \\
$\begin{array}{l}\text { Postoperati } \\
\text { ve }\end{array}$ & & & & & \\
$\begin{array}{l}\text { follow-up } \\
\text { Groups } \\
\text { without }\end{array}$ & 0 & 5 & 22 & 8 & \\
recurrence \\
$\begin{array}{l}1-5 \quad \text { years } \\
\text { later }\end{array}$ & & & & & \\
$\begin{array}{l}\text { Recurrence } \\
\text { groups }\end{array}$ & 0 & 1 & 3 & 5 & \\
\hline
\end{tabular}

\section{DISCUSSION}

Fibrous dysplasia (FD) is a fibrous bone disease, in which bone marrows and cancellous bones are replaced by fibrous connective tissues and irregular lesions. Lesions and normal bones have no clear boundaries. A complete surgical resection is not easy to carry out. Meanwhile, facial deformities caused by surgery place a heavy psychological burden on patients. Clinicians have been plagued by the non-surgical treatment of such diseases. Currently, its pathogenesis is not clear. The study of Sands WA et al. revealed that the onset and development of FD are regulated by the cAMP signaling pathway engendered by GSA 
gene mutation and the bone morphogenetic protein (BMP) signaling pathway.(4,5) In addition, there is a view that FD is caused by an imbalance between osteolysis and bone formation.(6) As a member of the TGF-b superfamily, BMPs are considered to regulate physical and psychological factors in a stable internal environment such as cancer, in the way in which cell proliferation, migration, differentiation and apoptosis are mediated(7-10).

TWSG1 was first discovered in drosophilas with a length of $23.5 \mathrm{KDa}$. It is expressed in both the embryonic and developmental stages (11) TWSG1 combines with BMP2, 4, 7 and so on in the intercellular matrix, inducing mesodermal development. On pathology, it is primarily considered to promote BMPS expression in order to make the disease more aggressive; and meanwhile, it inhibits osteoblast differentiation. $(9,12,13)$ Through immunohistochemistry examination, this study finds that fibroblasts and osteoblastoma in FD widely and highly express TWSG1, while rare osteoblast, bone cells and other cells suggest that an increase in TWSG1 expression might lead to the formation of immature osteoblasts and mesenchymal cells in FD. In recent years, through studies on mice deficient in TWSG1 expression, a number of scholars confirmed that its results lead to the formation of deformities(12) such as parvignathism; indicating that jaw growth and development are closely linked with TWSG1.

TWSG1 is an important promoting factor in craniofacial development. Through studies on mice deficient in TWSG1 expression, Petryk A et al. found that the development of craniofacial structures (14) such as forebrains and upper and lower jawboneswere restricted in such mice. However, in high concentrations of TSG, the conduction of the BMP signaling pathway is enhanced. Thus, developmental abnormalities in local bones are 
enhanced. In the study of Gylfe AE et al., the expression of eleven susceptibility genes in the colon examined cancer through experiments.(15) They believe that TWSG1 exhibits the role of a tumor suppressor in colon cancer. As a result, we found that through immunohistochemical studies, TWSG1 expression increased significantly in craniomaxillofacial FD. In addition, by comparison between the recurrence and static groups, TWSG1 expression in the FD recurrence groups was significantly higher; and the difference was statistically significant. However, similarly,several patients in the static groups had a higher TWSG1 expression. It can be speculated that TWSG1 in tissues of the lesion in craniomaxillofacial FD plays a key role in regulation. It can be considered that the higher the TWSG1 expression, the higher the possibility of postoperative continued growth. FD patients might further define the extent of surgery through TWSG1 detection. However, to totally exclude the possibility that other factors, together with TWSG1, regulate the occurrence and development of FD, needs further studies due to the limitation of detecting TWSG1isone factor in the disease.

\section{CONCLUSION}

In summary, the results of this study suggest that high TWSG1expression may play an important role in the occurrence and development of FD. Its invasive mechanism and the pathological state of immature osteoblasts in FD are regulated by TWSG1expression. They are important biological indicators of poor prognosis in FD. However, its precise regulatory 
mechanisms needs to be further explored. Further research on the molecular mechanism of TWSG1 and BMPS in FD is expected to provide a theoretical basis for the subsequent non-surgical treatment of FD.

\section{ACKNOWLEDGEMENTS}

We thank Professor Shao Yisen in the First Affiliated Hospital of Nanchang University for providing clinical data and samples. This study was supported by grants from Project of National Natural Science Foundation of China (Grant No. 31140007 , 81472516), Natural Science Foundation of Shanghai (No. 14ZR1424200) and Shanghai Leading Academic Discipline Project (No. S30206).

\section{REFERENCES}

1. DM Chen, KJ Zhang, LQ Zhang, et al. Clinical Analysis of 34 cases of 
craniomaxillofacial fibrous dysplasia. J Oral Sci Res. 2011, 27(12):1070-1074.

2. D Graf, S Nethisinghe, DB Palmer, et al. The developmentally regulated expression of twisted gastrulation reveals a role for bone morphogenetic proteins in the control of T cell development. J Exp Med. 2002,196 (2):163-71.

3. IC Scott, IL Blitz, WN Pappano, et al. Homologues of Twisted gastrulation are extracellular cofactors in antagonism of BMP Signal. Nature. 2001,410 (6827):475-8.

4. WA Sands, TM Palmer. Regulating gene transcription in response to cyclic AMP elevation. Cell Signal. 2008, 20: 460-466.

5. J Kiss, B Balla, JP Kosa, et al. Gene expression patterns in the bone tissue of women with fibrous dysplasia. Am J Med Genet A. 2010, 152A (9):2211-2220.

6. Li Enchao. Expression and clinical significance of BMP 2 and IL-6 in poor bone fibrous structures. Chinese J Bone Tumor Bone Dis. 2005, 4(3): 162-166.

7. JT Buijs, $\mathrm{G}$ van der Horst, $\mathrm{C}$ van den Hoogen, et al. The BMP2/7 heterodimer inhibits the human breast cancer stem cell subpopulation and bone metastases formation. Oncogene. 2012,31: 2164-2174.

8. A Singh, RJ Morris. The Yin and Yang of bone morphogenetic proteins in cancer. Cytokine Growth Factor Rev. 2010, 21: 299-313.

9. JE Sotillo Rodriguez, KC Mansky, ED Jensen, et al. Enhancedosteoclastogenesis causes osteopenia in twisted gastrulation-deficient mice through increased BMP signaling. J Bone Miner Res. 2009, 24: 1917-1926. 
10. IG Koutlas, CL Forsman, S Kyrkanide, et al. Autosomal dominant mesomandibular fibro-osseous dysplasia:a self-resolving inherited fibro-osseous lesion of the jaws. Front Physiol, 2012, 3: 458.

11. D Graf, PM Timmons, M Hitchins, et al. Evolutionary conservation, developmental expression, and genomic mapping of mammalian Twisted gastrulation. Mamm Genome. 201112:554-560.

12. M Oelgeschläger, J Larraín, D Geissert, et al. The evolutionarily conserved BMP-binding protein Twisted gastrulation promotes BMP signalling. Nature. 2000, 405(6788):757-63.

13. M Zaidi. Skeletal remodeling in health and disease. Nat Med. 2007, 13: 791-801.

14. JE Sotillo Rodriguez, KC Mansky, ED Jensen, et al. Enhancedosteoclastogenesis causes osteopenia in twisted gastrulation-deficient mice through increased BMP signaling. Bone Miner Res. 2009, 24 (11):1917-1926.

15. AE Gylfe, R Katainen, J Kondelin, etal. Eleven Candidate Susceptibility Genes for Common Familial Colorectal Cancer. PLoS Genet .2013; 9(10). 